A $R$ R T TÍl C U U L

$\begin{array}{lllllllll}R & E & F & L & E & X & I & O & N\end{array}$

\title{
LA EDUCACIÓN PÚBLICA DEL DEPARTAMENTO DE BOLÍVAR DURANTE LA PRIMERA MITAD DEL SigLO XX
}

\author{
PUBLIC EDUCATION IN THE DEPARTMENT OF BOLIVAR DURING THE \\ FIRST HALF OF THE TWENTIETH CENTURY
}

Por: Muriel Vanegas Beltrán*

Recibido: 18 de febrero de 2017 - Aprobado: 5 junio de 2018

* Profesora Asociada Universidad de Cartagena - Instituto Inter-
nacional de Estudios nacional de Estudios
del Caribe. Doctora en Ciencias de la Educación. Magíster en Estudios del Caribe. Historiadora.

\section{RESUMEN}

El presente artículo estudia la Educación Pública del departamento de Bolívar durante la primera mitad del siglo XX, a fin de analizar la recepción e implementación de las reformas educativas en las regiones de provincia y su articulación con los municipios de la costa Caribe colombiana. Con base en Fuentes Primarias sustentadas en Leyes Educativas, en Informes de Directores de Instrucción Pública y de Ministros de Educación, se plantea un análisis de esta coyuntura en el que se ponen de relieve las tensiones por modernizar el sistema educativo desde el nivel nacional, en contraste con las precarias condiciones sociales y económicas que imperaban en el departamento en este contexto. Se examinará, en consecuencia, las actuaciones de las autoridades educativas locales de acuerdo a las disposiciones normativas que se emanaban desde el nivel nacional.

Palabras clave: Reforma educativa, liberalismo, modernización, Bolívar, Caribe colombiano.

\begin{abstract}
This articles studies the public education in the department of Bolívar during the first half of the twentieth century seeking to analyze the reception and implementation of the education reforms in the regions provinces and towns of the Caribbean coast. Based on primary sources, particularly, legislation, and reports of the directors of Public Instruction and the Ministry of Education, it proposes an analysis of the conflictive national context in which the modernization of the educative system took place, and the precarious social and economic conditions that prevailed in the department in the meanwhile. This article will examine the local authorities' actions considering the norms emanating by authorities at the national level.
\end{abstract}

Keywords: Educational reform, liberalism, modernization, Bolivar, Colombian Caribbean.

\section{Introducción}

n América Latina, el siglo XX representó la aceleración de una serie de
transformaciones de índole social, política y económica que venían
gestándose al interior de las nacientes repúblicas, tras sus procesos 
independentistas (Henderson, 2006: 23-24). Dichos cambios se vieron impulsados por apuestas reformistas y de "modernización", que centralizaron el papel de los Estados, al tiempo que se involucraban las masas sociales como vehículo de legitimación ideológica de las agendas estatales (Urrego, 2015; Ossenbach, 2001: 32). Es en esta coyuntura en donde surge la apuesta por la educación pública para reforzar la productividad y generar cambios económicos con personas instruidas y letradas. Con ello se respondía al propósito de preparar los técnicos y obreros necesarios dentro de las emergentes industrias, así como los campesinos que demandaba la agricultura tecnificada. Este ambiente reformista fue el punto de partida para garantizar la verdadera apertura a los mercados internacionales en vertiginoso ascenso, y en ello, la educación pasaría a jugar un papel decisivo. (RHELA, 2018; Duarte, 2003).

Así, Argentina, Chile, México, Brasil, entre otros países, encarnan modelos educativos más representativos a nivel latinoamericano, pues asumieron un papel rector en el desarrollo de medidas reformistas, indistintamente de las orientaciones ideológicas que profesaran sus diversos gobiernos (Lobato, 2010; Serrano, Ponce de León y Rengifo, 2013; Tanck de Estrada, 2010; Chagas de Carvalho, 1998). Colombia no se vio ajena a este contexto y también impulsó decisiones políticas dirigidas a la transformación social en sintonía con los procesos de modernización de las naciones en proceso de industrialización. Como parte de este escenario mundial, el presente artículo aborda la Educación Pública ${ }^{1}$ del departamento de Bolívar en la primera mitad del siglo XX, iniciando el análisis desde el panorama nacional, para luego examinar la proyección regional de las reformas educativas conservadoras y liberales. El propósito fundamental es develar las limitaciones que subyacen en la búsqueda de la democratización de la educación a nivel nacional, según sus efectos y articulación con algunos municipios del Caribe colombiano, en medio de las precarias condiciones imperantes en su realidad política, económica y social.

\section{Los primeros esfuerzos por modernizar la educación en colombia}

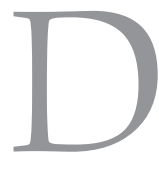

esde los umbrales del siglo XX colombiano, se emitieron importantes

leyes que aspiraban a la modernización de la educación nacional. La primera fue la Ley Orgánica de Educación 39 de 1903, y el decreto que la reglamentó en el siguiente año, acogió dicho propósito. Dictada bajo la presidencia de José Manuel Marroquín, esta ley fue el fundamento jurídico del sistema educativo nacional durante casi toda la primera mitad de dicho siglo. Con esta ley "se reafirmó el control religioso sobre la enseñanza, la separación
1 Para los fines del presente estudio, se tomará como objeto central de análisis la educación "oficial" o "pública", entendida esta como la que es planeada, financiada, inspeccionada y regulada por el Estado, de modo que la enseñanza "privada", suministrada por particulares u órdenes religiosas, no constituirá una categoría sustancial de valoración sino únicamente de referencia cuando se requiera, en función del tema central o contexto de la investigación. 
entre educación urbana y rural y la existencia de escuelas divididas en razón del sexo; se creó el Ministerio de Instrucción y Salud Pública y se recomendó la instrucción de asignaturas que propiciaran la formación técnica y práctica de los escolares" (Ministerio de Educación Nacional, 1959: 67). Igualmente, esta ley fijó un Sistema de Inspección nacional, departamental y local, dividió la educación en Primaria, Secundaria, Industrial y Profesional y subdividió la secundaria en Técnica y Clásica. Así mismo, con esta ley se ordenó que la Enseñanza Primaria Rural hiciera énfasis en las materias que "puedan habilitar a los ciudadanos para las tareas de la agricultura, la industria y el comercio" (Jaramillo, 1980: 281). De igual modo, se fijaron los gastos de la Educación Profesional, Secundaria y Normalista a la nación, los de Primaria a los departamentos y se autorizó a estos para que instituyeran estudios secundarios y de enseñanza profesional en caso de contar los recursos fiscales suficientes. Esta primera norma educativa del siglo XX continuó con un marco de orientación que seguía lo establecido por la Constitución de 1886 y en materia de la preeminencia de la religión católica en la enseñanza, en la aspiración a la unidad nacional y en el fomento de la riqueza como una de las metas del sistema escolar, no generó sustanciales cambios (Helg, 2001).

Es con la Ley 56 de 1927, que se hizo obligatoria la Enseñanza Primaria. Esta Ley también precisó las primeras disposiciones para promover una segunda reforma educativa de alcance nacional, en el contexto de los gobiernos conservadores. De esta manera, las Escuelas Normales existentes fueron cerradas con el objeto de reestructurarlas y darles una nueva orientación. Se crearon las Facultades de Educación y se unificó la Educación Primaria, Rural y Urbana. Igualmente, se decretó la libertad de escuela, se reestructuró el Ministerio de Instrucción y se especificaron las funciones relativas a la educación y a la salud, por lo cual, en adelante se denominaría Ministerio de Educación. Este cambio lo liberó de compartir recursos presupuestales con los gastos de beneficencia y leprosería. Sin embargo, buena parte de estas modificaciones, tendrían que esperar algunos años para ser introducidas en el ámbito educativo nacional.

Exceptuando el Decreto 1487 de 1932, las políticas educativas del presidente liberal Enrique Olaya Herrera, (1930-1934), fueron una extensión de las agenciadas por los gobiernos conservadores predecesores. Dicho decreto, expedido un 13 de septiembre, oficializaba una reforma a la Enseñanza Primaria y Secundaria que, entre otros aspectos, establecía la obligatoriedad de la Instrucción Primaria tanto en las escuelas urbanas como en las rurales 
y los requisitos para aspirar al nivel de bachillerato, al universitario y a la carrera del magisterio. Así mismo, por lo reglamentado en dicho decreto solo en los colegios oficiales de segunda enseñanza se podía acceder al grado de bachiller o en los colegios privados que obtuvieran esta facultad del Ministerio de Educación.

La memoria del Ministro de Educación Nacional presentada al Congreso de 1933 por Julio Carrizosa, inicia su introducción aduciendo:

"Se ha sostenido entre nosotros, no por pocas personas, que el problema de la reforma instruccionista es un problema legislativo; otros han dicho que tal problema se resuelve solamente con dinero, y no han faltado quienes afirmen que el problema consiste, simplemente, en una acertada labor administrativa. Salta a la vista de quien estudie con algún detenimiento esta cuestión, que ninguna de las opiniones anteriores es completamente cierta, pues la resolución del problema instruccionista participa al mismo tiempo de todos estos aspectos: tiene de reforma legal, consiste también en una acción administrativa, y requiere naturalmente dinero. Tal es la razón de su gran complejidad aquí y en otras partes, ya que puede decirse que todos los Estados modernos padecen el problema no resuelto de la educación pública. Es necesario, por lo tanto, repasar todos los aspectos de esa empresa instruccionista del Estado, para señalar sus deficiencias y determinar las causas de esas deficiencias" (Ministerio de Educación Nacional, 1933: 4).

A pesar de estas iniciativas, el campo educativo durante el cuatrienio de Olaya, no reveló modificaciones estructurales y la política educativa quedó en manos de ministros conservadores. Un movimiento de reformas de mayor envergadura se hacía cada vez más apremiante (Acevedo y Villamizar, 2015; Herrera y Díaz, 2001). Según María Teresa Calderón e Isabela Restrepo “el verdadero cambio político sólo comienza en 1934 con la elección de Alfonso López Pumarejo, figura central del Partido Liberal desde tiempo atrás. Es el que marca la verdadera transformación y convoca a una nueva generación de dirigentes, algunos de los cuales habían coqueteado con ideas socialistas, dispuestos a modernizar a Colombia" (Calderón y Restrepo, 2010: 57).

Bajo el gobierno liberal de López Pumarejo (1934-1938) se implementó el Acto Legislativo No 1 del 5 de agosto 1936, reformatorio de la Constitución, que reza en su Art. 14: "Se garantiza la libertad de enseñanza. El Estado tendrá, 
sin embargo, la suprema inspección y vigilancia de los institutos docentes, públicos y privados, en orden a procurar el cumplimiento de los fines sociales de la cultura y la mejor formación intelectual, moral y física de los educandos. La enseñanza primaria será gratuita en las escuelas del Estado, y obligatoria en el grado que señale la ley" (Senado de la República de Colombia. Diario Oficial $N^{\circ} 23263$ de 22 de agosto de 1936: 1). Se trataba, en esencia, de una Reforma Constitucional y con dicho parágrafo se normatizó la injerencia estatal en la educación nacional y se prohibió la discriminación educativa en razón de sexo, raza, religión o nacimiento ilegítimo.

Más adelante, con la aprobación de la Ley 257 de 1938 se autorizó al gobierno a celebrar contratos para nacionalizar institutos de Enseñanza Secundaria, departamentales, municipales o privados. El mandato de Eduardo Santos (1938-1942) no introdujo sustanciales modificaciones pues las políticas que se implementaron se limitaron a reglamentar la legislación vigente y a buscar crecimientos "cuantitativos" (Santos, 1939: 39-43).

Durante este gobierno, Jorge Eliécer Gaitán como Ministro de Educación (1940-1941) presentó un plan de nacionalización de la Enseñanza Primaria. La medida consistía, básicamente, en eximir a los departamentos y municipios de toda responsabilidad educativa y concentrarla en el gobierno central. En el informe que presentó a las Cámaras Legislativas en 1940 expone sus criterios sobre la nacionalización de la enseñanza con lo siguiente sustentos:

"En el funcionamiento de la escuela pública colombiana hay una verdadera sociedad anónima formada por la nación, el departamento y los municipios, que se debate en medio de la incongruencia". Allí todos son responsables y ninguno tiene la responsabilidad"... "La nación, de acuerdo con la anarquía reinante debe proveer de útiles a las escuelas, el departamento ha de pagar los maestros, y a los municipios les toca ofrecer los locales"..."En ocasiones es el departamento el que ofrece los maestros, porque para ello tiene partidas en su presupuesto, pero al municipio le falta lo necesario para hacer entrega de locales. Resultado, que no habrá escuela. En otras el fenómeno es a la inversa de lo aquí enunciado. Total, que tampoco habrá escuela. En veces faltan muebles, pero habrá edificio. Total, que no habrá escuela. Las más de las ocasiones habrá maestros y locales, pero los municipios están sin recursos para el transporte de los útiles que la nación facilita y tiene. Total, no funcionará la escuela" (Gaitán, 1940: 20-21). 
Sin embargo, el rechazo de tal propuesta por parte del Congreso debido a la oposición de liberales y conservadores, sepultó la iniciativa y llevó a Gaitán a presentar renuncia. Tampoco se logró modificar la legislación que existía desde 1903 según la cual, el cuidado y manejo de la Educación Primaria recaía sobre los departamentos y municipios. Germán Arciniegas, Ministro de Educación de 1942 a 1946, criticó dicha legislación que, a su modo de ver, origina: "escuelas sin higiene, sin luz, aire ni espacio físico, por la pobreza fiscal de los municipios y el mal uso de los dineros municipales destinados a la educación" (Arciniegas, 1942: 38). Sin duda, la aplicación de las reformas para modernizar la educación careció de condiciones materiales y financieras adecuadas para lograr sus objetivos.

De la nación a la región: la educación pública de Bolívar en tiempos de "modernización"

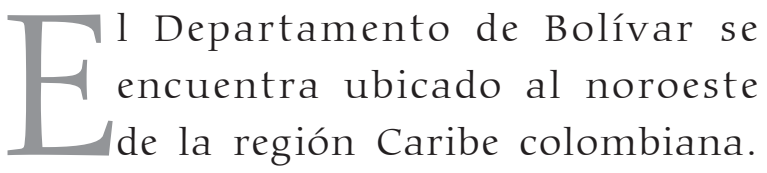
Fue creado por la Constitución de la República de Colombia de $1886^{2}$. Desde el período Colonial comprendió, además del actual departamento de Bolívar, los actuales territorios de los departamentos de Atlántico, Sucre y Córdoba, los cuales le fueron desagregados en 1905, 1951 y 1966 respectivamente. Por tanto, durante el período del que el presente estudio se ocupa, tanto Sucre como Córdoba, y sus

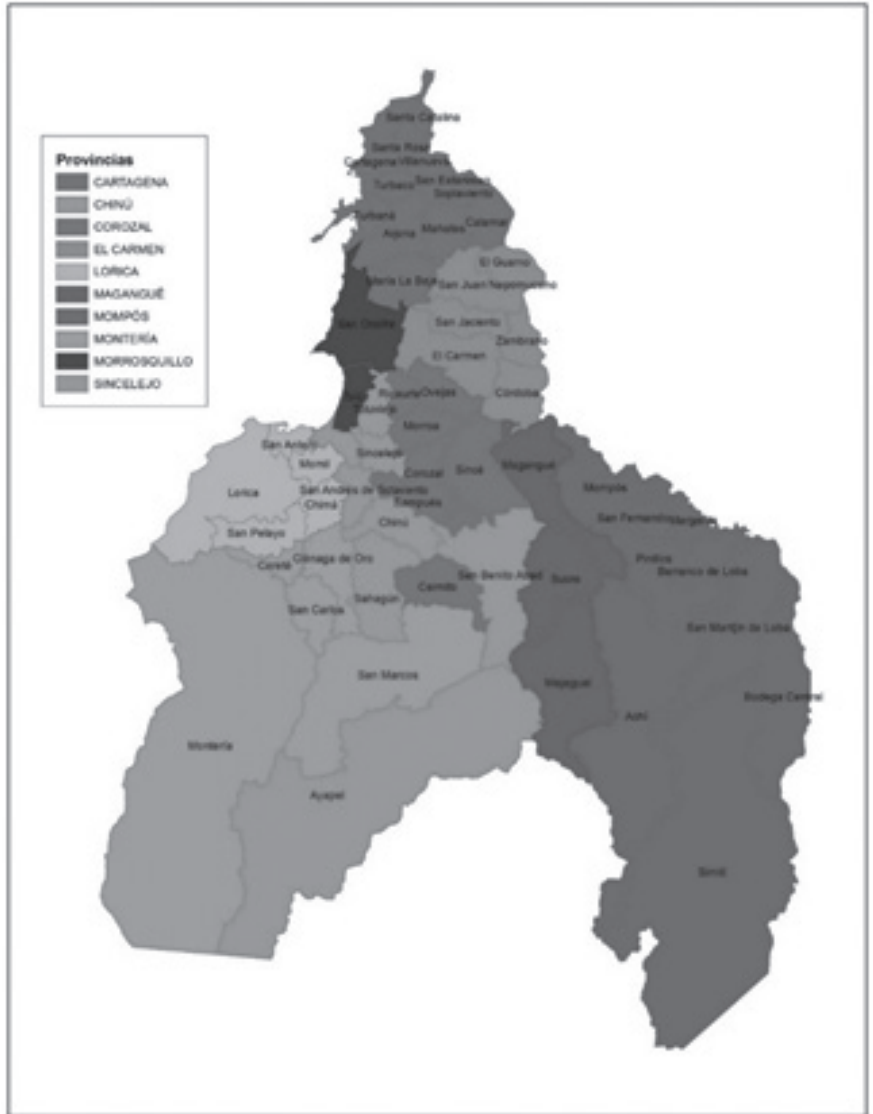

Departamento de Bolívar, 1905-1951

Fuente: Elaboración propia respectivos municipios, le correspondían todavía a Bolívar geográfica y políticamente.

Bolívar tenía una superficie de $32.070 \mathrm{Km}^{2}$, posicionándose como el tercer departamento con mayor tamaño después de Antioquia y Boyacá, y el cuarto con mayor número de habitantes, después de Antioquia, Cundinamarca y Caldas (DANE, 1938).

2 Hasta 1886, la división política de Colombia se basaba en estados. A partir de ese año, el Consejo Nacional de Delegatarios dispuso cambiar el nombre que tenía el país (Estados Unidos de Colombia) por el de República de Colombia y el término departamentos sustituyó el de estados, pasando a depender del poder central, pero conservando los mismos límites geográficos que tenían previamente. 


\section{Instrucción primaria}

El número de escuelas que funcionaron en Bolívar en 1920 fue de 279, distribuidas así:

\section{Número y tipo de escuelas primarias en Bolívar, 1920}

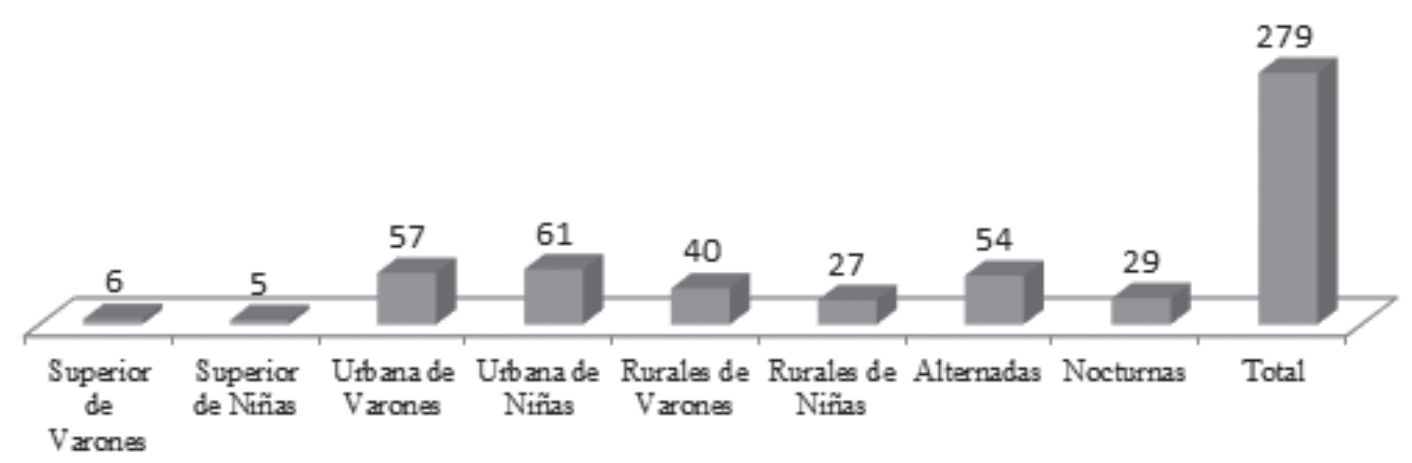

Fuente: Elaboración propia a partir del Informe del Director General de Instrucción Pública al Gobernador del Departamento de Bolívar. 1921: 4.

Como puede apreciarse, existían muy pocas Escuelas Superiores y la mayoría de las escuelas se encontraban en el ámbito urbano. El sector rural participaba con el 17\% del total de escuelas en el departamento, con lo cual se presenta una carencia considerable de centros educativos en las zonas rurales de Bolívar donde habitaba la mayoría de la población a inicios de la década de 1920. En el informe que el Director General de Instrucción Pública, Nicomedes Flórez, presentó al Gobernador de Bolívar en 1921, se pone de manifiesto que pese a las disposiciones oficiales de dividir la instrucción en Primaria, Secundaria, Profesional e Industrial, no existía en el departamento ningún establecimiento de la última clase, pues aunque la Ordenanza Número 57 de 1917 dispuso la fundación de Escuelas Industriales en varias provincias del departamento, "tal disposición ha venido a ser letra muerta", cuando dejó de establecerse en el presupuesto las partidas apropiadas para llevar a cabo el funcionamiento de las mismas. (Informe del Director General de Instrucción Pública al Gobernador del Departamento de Bolívar, 1921: 3).

\section{Instrucción secundaria y profesional}

La Instrucción Secundaria oficial se atendía en el departamento en dos colegios: la llamada Facultad de Filosofía y Letras de la Universidad de Cartagena, donde cursan estudios de Bachillerato los varones, y el Colegio de Nuestra Señora del Carmen para señoritas, además de las Escuelas Normales (y en sus respectivas Escuelas Anexas como centro de prácticas). La Enseñanza 
Profesional se impartían en dos facultades de estudios superiores existentes en la Universidad de Cartagena: la de Derecho y Ciencias Políticas y de Medicina y Ciencias Naturales ${ }^{3}$.

\section{Escuelas normales}

En 1920 se registraron 41 alumnas matriculadas, "con dos retiradas por enfermedad comprobada" (Informe del Director General de Instrucción Pública al Gobernador del Departamento de Bolívar, 1921: 31). La Escuela Anexa a la Normal de Varones funcionó con un personal de 108 alumnos matriculados y la Escuela Anexa a la Normal de Niñas tuvo 107 matrículas. En ambos casos, con un promedio de asistencia de 80. Según el Informe de la Escuela Normal Nacional de Institutoras de Cartagena:

"Los locales donde funcionan las escuelas no tienen todas las condiciones que requieren los edificios destinados a institutos de esa naturaleza, por no haber sido construidos a ese efecto, sin embargo, el de la Escuela de Varones es bastante capaz y posee buenos dormitorios y suficiente número de aulas para el dictado de las clases, pero el salón destinado a la Escuela Anexa es inadecuado: el techo del salón es bajo, no tiene la suficiente luz, y es ya estrecho para el número de alumnos que concurren a ella. El local de la Normal de Señoritas adolece también de muchas falencias...careciendo, además, de la ventilación requerida. No se dispone tampoco de una pieza para enfermería..." (Informe del Director General de Instrucción Pública al Gobernador del Departamento de Bolívar, 1921: 29).

Los inconvenientes apuntados fueron difíciles de subsanar debido a las limitaciones para conseguir locales que reúnan las condiciones higiénicas y pedagógicas que requieren este tipo de planteles. El encargado de esta Dirección estima muy apropiado que la próxima Asamblea dicte una ordenanza autorizando a la Gobernación del Departamento para contratar un empréstito destinado a la construcción de dos locales adecuados para dichas escuelas. Sin embargo, estas iniciativas no fueron acatadas por las autoridades correspondientes (Informe del Director General de Instrucción Pública al Gobernador del Departamento de Bolívar, 1921: 30).

\section{Universidad de Cartagena}

Manuel Dávila Flores, rector de la Universidad de Cartagena informó al Gobernador de Bolívar que para 1920 la matrícula se componía de 305

3 Para un análisis profundo del funcionamiento de la Universidad de Cartagena en este contexto ver: PIÑERES, Dora. (2008). Modernización, universidad y región. El caso de la Universidad de Cartagena, 19201946. Cartagena: Universidad de Cartagena Universidad Pedagógica y Tecnológica de Colombia (UPTC), RUDECOLOMBIA. 
alumnos, en su mayoría vinculados a la Facultad de Filosofía y Letras, seguidos de la Facultad de Medicina y el más bajo índice de las matrículas correspondía a la Facultad de Derecho y Ciencias Políticas, según se observa en la siguiente tabla:

Alumnos matriculados en la Universidad de Cartagena, 1920

\begin{tabular}{|c|c|}
\hline \multicolumn{2}{|c|}{ UNIVERSIDAD DE CARTAGENA } \\
\hline UNIVERSIDAD DE CARTAGENA & ALUMNOS MATRICULADOS EN 1920 \\
\hline Facultad de Filosofía y Letras & 235 \\
\hline Facultad de Derecho y Ciencias Políticas & 28 \\
\hline Facultad de Medicina y Ciencias Naturales & 42 \\
\hline TOTAL & 305 \\
\hline
\end{tabular}

Fuente: Elaboración propia a partir del Informe del Rector de la Universidad de Cartagena, 1921. p.50.

La financiación de la Educación Secundaria seguía siendo deficiente para sufragar los gastos de una educación adecuada, lo que impedía aumentar la oferta educativa en otras provincias y que esta se concentrara en Cartagena, la ciudad capital. En términos de gastos e inversiones, en la Instrucción Secundaria el monto total llegaba a los \$ 53.092 distribuidos como aparece en la siguiente tabla gráfica:

\section{Gastos en la instrucción secundaria de Bolívar, 1920}

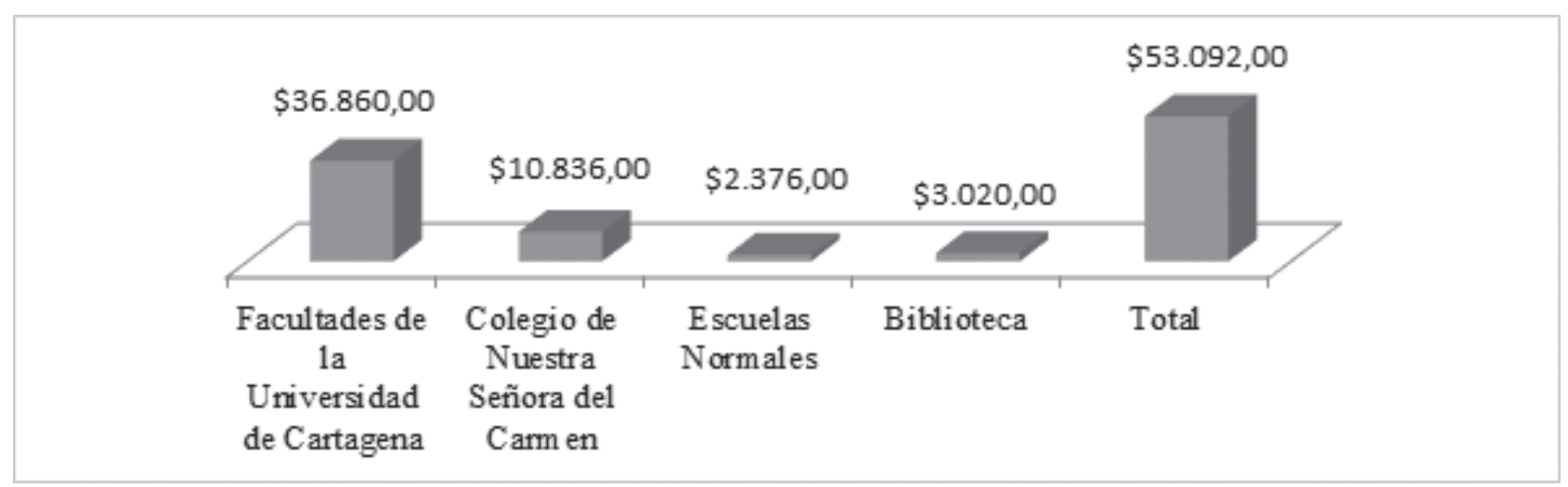

Fuente: Informe del Director General de Instrucción Pública al Gobernador del Departamento de Bolívar, $1921: 21$.

La Universidad de Cartagena con sus tres facultades recibía más del 52\% de los recursos destinados al ramo; el Colegio de Nuestra Señora del Carmen, con 176 alumnos incluida su Escuela Anexa, recibía el 22\% del presupuesto 
seguida de la Biblioteca que percibía el 10\%. El 6\% restante se invertía en las Escuelas Normales que se presume que era un valor demasiado inferior al que necesitaban estas escuelas para lograr fortalecer el sistema educativo en el departamento.

Como se puede advertirse, al iniciar la década de 1920, la educación en el departamento era muy limitada para satisfacer la demanda de sus provincias donde evidentemente se presentaba un aumento de la población en edad escolar y donde el desarrollo de las zonas seguía aplazado. No obstante, al finalizar esta década se evidencia un robustecimiento del sistema educativo en el departamento que puede explicarse en un aumento en la inversión y un incremento en instituciones educativas, de estudiantes y profesores.

Entrando en la década de 1930, el informe que rinde Rafael Ricardo hacia 1931, como Director de Educación Pública del departamento al Gobernador de Bolívar, señala:

"Un pueblo que apenas va por el alfabeto, está en la infancia de su historia. Cuando alimentado nuestro espíritu por el más sano y noble optimismo, traspasamos los umbrales de aquel remedo de casa habilitada para la escuela, casa en cuya construcción se ingenió el arquitecto improvisado por levantar el Palacio del hombre de la edad de piedra; cuando nuestra vista se posa en aquella legión de niños haraposos, descalzos, sucios, tristes, aureolados por una amargura que cual pesado fardo agobia sus cabecitas inocentes; cuando al frente de la clase observamos al pobre toma-lecciones, mal vestido porque está mal pagado, para quien la pedagogía es una palabra de extraño significado, que su mente aletargada apenas alcanza a comprender, se nos estruja el alma" (Ricardo, 1931: 4).

Llama la atención como resultan reiterados los pronunciamientos de las autoridades educativas sobre las precarias condiciones de las escuelas, de los estudiantes y de los maestros, evidenciándose con ello la falta de atención por parte de las autoridades centrales y departamentales. De acuerdo con el Art. $9^{\circ}$ de la Ley 39 de 1903, y el 43 del Decreto No 491 del poder ejecutivo nacional, como de otras disposiciones departamentales, corresponde a los municipios obligatoriamente suministrar locales y mobiliarios para las escuelas. A pesar de ello, "este es un punto sumamente importante que no todos los Municipios han sabido cumplir. Las condiciones pedagógicas de los 
locales son completamente desconocidas por el ambiente municipal, en donde se cree que cualquier casa de familia puede servir para escuela, lo que es un enorme error" (Hernández, 1932: 4), afirma José V. Hernández, Director de Educación Pública de Bolívar en 1932, a lo que, además, agrega:

“Por ministerio de la Ley y Ordenanzas reglamentarias de la Educación popular, los municipios están obligados a suministrar el local para la escuela y el mobiliario respectivo. Por inveterada costumbre que se debe corregir, los municipios ponen poco interés en favor de la educación y dejan al departamento la totalidad de las iniciativas en este sentido. Cartagena rica y próspera ciudad, cuyo presupuesto general de gastos sube a la considerable suma de más de un millón de pesos, dedica \$35.000 a la educación, es decir, un 3,3\% de su presupuesto. En igualdad de circunstancias están los demás municipios del departamento" (Hernández, 1932: 5).

De esta manera se advierte que locales sin ninguna comodidad, sin condiciones pedagógicas y de malos materiales salen costando cantidades muy superiores al valor que realmente representan, y aunque la Dirección es la que tiene en última instancia la facultad de aprobar o desaprobar tales contratos, se encuentra en la imposibilidad muchas veces de tener conocimiento cierto de las desventajas que ellos encierran, o al contrario, se le informa de que los contratos son ventajosos y en esa creencia los aprueba. Las disposiciones expresas determinan que los locales de las escuelas deben hacerse de acuerdo a planos que llenan determinados requisitos (de ubicación, amplitud, comodidad, higiene, entre otros), aprobados por el gobierno, pero como en los municipios se va construyendo o adquiriendo por compras de lenta consecución por los señalados manejos burocráticos y administrativos, no es posible tener una mirada precisa sobre este particular. Lo que sí queda claro es que tales normativas no son aplicadas en el territorio bolivarense.

\section{Implementación de la reforma educativa liberal, 1936-1946}

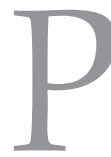
ara 1938, la población total de Bolívar era de 765.194 habitantes, de los cuales 379.062 eran hombres y 386.132 eran mujeres, lo que significa que había 102 mujeres por cada 100 hombres. De esos 765.194 habitantes, el 22\% representaba la población en edad escolar, es decir, entre 7 y 14 años $^{4}$, lo que equivalía a 168.739 personas. De este número, solo 49.034 era población alfabeta, frente a 119.702 analfabeta, representando el 29,1\% y 
el 70.9\%, respectivamente. De esas 119.702 personas analfabetas, 53\% eran hombres y 47\%, mujeres (DANE, 1938).

Otro aspecto de crucial importancia es el desequilibrio que presenta la instrucción entre la parte urbana y rural de los municipios. El siguiente cuadro revela la desproporción entre los analfabetos urbanos y rurales:

\section{Población alfabeta y analfabeta en edad escolar, urbana y rural por departamentos, 1938}

\begin{tabular}{|c|c|c|}
\hline \multirow{2}{*}{ DEPARTAMENTOS } & URBANA & RURAL \\
\cline { 2 - 3 } & ANALFABETOS DE EDAD ESCOLAR & ANALFABETOS DE EDAD ESCOLAR \\
\hline Antioquia & 18.838 & 94.613 \\
\hline Atlántico & 23.340 & 6.495 \\
\hline Bolívar & 31.830 & 87.997 \\
\hline Boyacá & 6.538 & 96.171 \\
\hline Caldas & 15.332 & 62.258 \\
\hline Cauca & 3.573 & 45.518 \\
\hline Cundinamarca & 25.911 & 91.169 \\
\hline Huila & 5.898 & 24.557 \\
\hline Magdalena & 14.258 & 33.112 \\
\hline Nariño & 7.637 & 49.920 \\
\hline Norte de Santander & 7.808 & 38.754 \\
\hline Santander & 9.439 & 70.015 \\
\hline Tolima & 10.993 & 66.324 \\
\hline Valle del cauca & 14.870 & 41.837 \\
\hline TOTAL & $\mathbf{1 9 6 . 2 3 9}$ & $\mathbf{8 0 8 . 7 9 8}$ \\
\hline
\end{tabular}

Fuente: DANE, Censo de 1938.

Obsérvese como entre los departamentos del país figura Bolívar con la alta cifra de 31.830 analfabetos en edad escolar en la parte urbana, lo que corresponde a un 26,6\% (cifra más elevada en comparación con las del resto de departamentos), y 87.997 analfabetos en edad escolar en las áreas rurales, es decir, un 73,4\%. También resultan alarmantes las cifras de los departamentos de Boyacá, con un 93,6\%; Cauca, con un 92,7\%; Santander, con un 88,1\%, Nariño, con un 86,7\%; Tolima, con un 85,8\% y Antioquia, con un 83,4\%, con los casos más elevados de analfabetismo rural.

4 Para esta época, Colombia, Argentina, Chile, Bolivia y Canadá partían de la población mayor de 7 años para el estudio del analfabetismo; en cambio, Estados Unidos, Méjico y Cuba, parten de la población mayor de 10 años. 
A nivel departamental, las declaraciones de Luis A. Galofre, Director de Educación de 1936 en su informe al Gobernador, Manuel F. Obregón revelaba serias precariedades en las condiciones y funcionamiento de las escuelas de Bolívar, que demandaban mayor atención y urgente intervención por parte de las autoridades regionales y nacionales:

"Da grima contemplar, por ejemplo, el estado en que se encuentra el 90\% de los mobiliarios de nuestras escuelas. Pocas son aquellas en que todos los niños pueden sentarse cómodamente, y muy contadas las que tienen bancos pedagógicamente acondicionados. La enorme mayoría carece de todo. Yo he conocido algunas en que no hay siquiera una silla para el maestro. Alarma la carencia de materiales para la enseñanza, En el caso de Geografía, no hay escuela rural ni urbana que tenga un mapa" (Informe del Director de Educación Pública al señor Gobernador del Departamento, 1936: 3).

"Las necesidades de la educación pública en Bolívar son tan numerosas, que me abruma el temor de hacerme demasiado prolijo en su exposición...tales deficiencias no son nuevas y la gestión oficial, en más de una ocasión, ha incidido en los extremos lamentables a que ellas llegan. Todo está por hacer en este ramo". (Informe del Director de Educación Pública al señor Gobernador del Departamento, 1936: 5).

Al tratar la Instrucción Primaria, llama la atención sobre dos factores importantes: locales y maestros. Los primeros "en malas condiciones de aire y luz", y los segundos, "mal pagados y remunerados sin puntualidad. Pero empiezan a capacitarse: hay en 1936, 70 jóvenes de ambos sexos preparándose en las mejores normales del país con becas nacionales y están por adjudicarse 35 más" (Informe del Director de Educación Pública al señor Gobernador del Departamento, 1936: 13).

En 1938 funcionan en el departamento 463 escuelas, y en el año escolar anterior asistieron 19.907 de 32.323 alumnos matriculados, lo cual no representa, en concepto del Director Departamental de Educación, ni siquiera la mitad de la población escolar: "En la conferencia de directores que tuvo lugar en Bogotá el año pasado (1937) se evidenció que la escuela colombiana no recibe la totalidad de los escolares, que es de un millón y medio. Solo un millón puede recibir el beneficio de la educación: Corresponde a las Secciones 
ir cubriendo ese saldo en cuanto lo permitan sus presupuestos", afirma el Director Miguel Ángel Royo. (Informe del Director de Educación Pública al señor Gobernador del Departamento, 1936: 7). El gobierno nacional ha auxiliado a las escuelas suministrándole abundante material pedagógico, que ha facilitado la tarea de los maestros. Pero la obra educativa sigue desarrollándose en edificios inadecuados y las escuelas siguen adoleciendo de mobiliario conveniente. Sin embargo, se avanzó con el Decreto Ejecutivo No 888 de 1937 aumentándose con él la cuota nacional y departamental destinada a la construcción y adecuación de edificios escolares.

Adicionalmente, las distintas escuelas de Bolívar eran atendidas por un alto número de maestros sin grado. Para empezar a subsanar esta situación, en 1938 se empiezan a asignar becas departamentales para avanzar en la formación de los nuevos maestros que demandaba la región. La siguiente taba discrimina los 39 becarios favorecidos y las ciudades e instrucción a las que fueron enviados para profesionalizarse:

Número de becas departamentales, 1938

\begin{tabular}{|c|c|c|}
\hline CIUDAD & INSTITUCIÓN & NÚMERO DE BECARIOS \\
\hline Bogotá & Colegio San Bartolomé & 1 \\
\hline Bogotá & Escuela Nacional de Bellas Artes & 1 \\
\hline Bogotá & Enseñanza Especializada & 1 \\
\hline Bogotá & Gimnasio Moderno & 2 \\
\hline Medellín & Escuela Normal de Medellín & 1 \\
\hline Medellín & Colegio de San Ignacio de Medellín & 1 \\
\hline Barranquilla & Instituto Ariano & 1 \\
\hline Mompox & Colegio de Pinillos de Mompox & 20 \\
\hline Sincelejo & Instituto de Sabanas de Sincelejo & 1 \\
\hline Cartagena & Universidad de Cartagena & 8 \\
\hline Cartagena & Instituto Comercial de Cartagena & 1 \\
\hline Cartagena & Colegio de Santa Teresita & \\
\hline
\end{tabular}

Fuente: Informe del Director General de Instrucción Pública al Gobernador del Departamento de Bolívar, 1938.

Analizando la proporción que ilustra la tabla anterior, a la ciudad de Bogotá se enviaron cuatro becarios, cada uno de ellos a las siguientes instituciones: Colegio San Bartolomé, Escuela Nacional de Bellas Artes, Enseñanza Especializada y Gimnasio Moderno. En Cartagena se capacitaron diez becarios, 
ocho de ellos en el Instituto Comercial, uno en la Universidad de Cartagena y otro en el Colegio de Santa Teresita. Dos becarios se enviaron a Medellín, uno de ellos a la Escuela Normal y otro al Colegio San Ignacio de esta ciudad. Barranquilla y Mompox recibieron cada una un becario. Sincelejo fue la cuidad con el mayor número de becarios admitidos, recibiendo el 75\% de las becas que se adjudicaron a Bolívar, otorgándose al Instituto Sabanas.

No obstante, para 1938, el Director de Educación, Miguel Ángel Royo, manifestaba las mismas condiciones precarias de la educación y de la situación laboral de los maestros en relación a su trabajo y a su remuneración, que se denunciaban en los años anteriores: "Quiero destacar dos necesidades de inaplazable urgencia: Aumentar generosamente el presupuesto de la educación y seleccionar sin debilidades un magisterio comprometido y capacitado" (Informe del Director de Educación Pública al señor Gobernador del Departamento, 1938: 5). Para esos años ya contaban el departamento con 670 maestros, de los cuales pertenecen 6 a la primera categoría; 45 a la segunda; 183 a la tercera y 463 a la cuarta. La Ley 2 de 1937 estableció un sueldo mínimo para los maestros, fijado en la suma de 40 pesos mensuales. El Ministerio de Educación Nacional procedió también con esta ley a establecer las categorías según las cuales quedó clasificado el personal docente y solo podían ser nombrados maestros de escuela aquellos que figuraran en el escalafón nacional. Los gobernadores, por su parte, debían ceñirse estrictamente a las categorías así establecidas.

Para finalizar, ciertamente el Estado, a través del Ministerio de Educación Nacional, imprimió a la educación una dirección oficial y lideró un discurso pedagógico desligado del religioso, pero su fragilidad le impidió asumir las funciones que le competían en correspondencia con una nación moderna, lo que significó realizaciones parciales de las reglamentaciones reformistas dispuestas. Este contexto, signado por fluctuaciones e inestabilidades nacionales e internacionales, representó una de las época más complejas de la historia contemporánea nacional, en el que los alcances de las reformas liberales fueron inferiores a las expectativas: La tasa de analfabetismo de hombres y mujeres mayores a siete años superaba el 50\% a fines de la década del cuarenta; muchas poblaciones carecían de escuelas y colegios; millares de colombianos, aun adultos, quedaban por fuera del sistema educativo sin posibilidades de acceder a la Educación Media o Superior, de tal suerte que las metas propuestas resultaron más ambiciosas que viables. 


\section{Conclusiones}

T os gobiernos conservadores y liberales que idearon e impulsaron las reformas educativas de la primera mitad del siglo $\mathrm{XX}$, realizaron Jesfuerzos importantes desde el punto de vista financiero para asumir los costos que medidas de esta envergadura requerían. El primer paso dado, implementado a nivel constitucional, fue el aumento del gasto del Estado destinado a la Educación Pública. Aun así, pese a que la ley 12 de 1934 normatizó que el 10\% del presupuesto nacional debía invertirse en educación, las partidas presupuestales ejecutadas realmente oscilaron entre el $7 \%$ y el 8\% (Helg, 2001: 150-152). Adicionalmente quedó demostrado que durante la primera mitad del siglo XX la Educación Pública es, estructuralmente y en esencia, una tarea de los departamentos. Sobre estos recae la mayor parte de la financiación, son los que nombran los maestros e inspeccionan los establecimientos educativos. Sin embargo, la articulación de la política educativa nacional, que regulaba el sistema y prescribía planes de estudio y normas pedagógicas, con los departamentos y municipios que designaban y pagaban a los maestros resultó limitada; la capacidad financiera de los departamentos era desigual y la mayoría carecía de los recursos necesarios para efectuar los pagos adecuados y oportunos de los maestros y asumir el mantenimiendo de los planteles educativos. A ello se añade que las escuelas oficiales carecían de recursos pedagógicos y los locales estaban en condiciones precarias, antihigiénicas, oscuros y con inapropiada ventilación Estas situaciones eran factor común en la mayoría de las zonas urbanas y rurales de las regiones, y las autoridades del departamento de Bolívar así lo registraron a lo largo del periodo estudiado.

Por supuesto, es de destacar que a lo largo del contexto analizado se idearon novedosas acciones en aras del fomento y mejora del sistema educativo nacional, de cara a la tendencia continental de desarrollar un intervencionismo estatal para dinamizar las industrias. No obstante, aun reconociendo sus importantes progresos en materia de concepción de la educación y de renovación del aparato educativo, tales reformas se plantearon de una manera tan generalizante que no se consideraron las realidades y particularidades de las distintas regiones del país. Sus efectos en el departamento de Bolívar arrojan resultados modestos y parciales que se evidencian, por una parte, en que el radio de acción alcanzado por el Ministerio de Educación Nacional en esta temporalidad se vio muy limitado por los escasos recursos financieros que se le asignaron. Ello revela que la capacidad política y económica del Estado resultó insuficiente a la hora de arrogarse las funciones inherentes a un estado 
moderno y, a su vez, de llevar al Caribe colombiano, y a sus provincias, las iniciativas de progreso y modernización nacional desde la educación, lo cual ha incidido en los rezagos regionales evidentes en tiempos presentes.

\section{REFERENCIAS BIBLIOGRÁFICAS}

\section{Fuentes primarias}

ARCINIEGAS, Germán. (1942). Ministro de Educación Nacional. Memoria 1942. Bogotá: Imprenta Nacional.

DANE. Censos de 1918, 1928, 1938, 1951.

GAITÁN, Jorge Eliécer. (1940). Obra educativa del gobierno en 1940. Bogotá: Imprenta Nacional.

HERNÁNDEZ, José V. (1932). Informe de educación pública de Bolívar. Cartagena: Ediciones de la Imprenta Departamental.

Informe de Directores Generales de Instrucción Pública a los Gobernadores del Departamento de Bolívar, 1920-1925.

MINISTERIO DE EDUCACIÓN NACIONAL. (1959). La educación colombiana, 1903-1958. Tomo I. Compilación Legislativa. Bogotá: Imprenta Nacional.

RICARDO, Rafael. (1931). Director de Educación Pública de Bolívar. Informe al señor Gobernador del Departamento. Cartagena: Imprenta departamental.

SANTOS, Eduardo. (1939). Mensaje del Presidente de la República de Colombia al Congreso Nacional en sus sesiones de 1939. Bogotá: Imprenta Nacional.

Senado de la República de Colombia. Diario Oficial No 23263 de 22 de agosto de 1936. Bogotá.

\section{Fuentes secundarias}

ACEVEDO, Álvaro y VILLAMIZAR, Carlos. (2015). “Discursos y prácticas culturales durante la 'Revolución en Marcha'. Reforma educativa y cambio social". En: Revista Ciencias Sociales y Educación, Vol. 4, № 7. Medellín: Universidad de Medellín, págs. 37-52.

CALDERón, María Teresa y RESTREPO, Isabela. (2010). Colombia 1910-2010. Bogotá: Taurus.

CHAGAS DE CARAVALHO, Marta. (1998). Molde nacional fôrma cívica: higiene, moral trabalho no projeto da Associação Brasileira de Educação (1924-1931). Bragança Paulista: EDUSF. págs. 2027. 
DUARTE, Jesús. (2003). Educación pública y clientelismo en Colombia. Medellín: Universidad de Antioquia,

HELG, Aline. (2001). La educación en Colombia: 1918-1957. Una historia social, económica y política. Bogotá, Serie Educación y Cultura, Universidad Pedagógica Nacional y Plaza \& Janés Eds.

HENDERSON, James. (2006). La modernización en Colombia. Los años de Laureano Gómez, 1889_ 1965. Medellín: Universidad de Antioquia.

HERRERA, Martha y DíAz, Carlos. (comp.). (2001). Educación y cultura política una mirada interdisciplinaria. Bogotá: Plaza y Janés editores, Universidad Pedagógica de Colombia.

JARAMILLO, Jaime. (1980). "El proceso de la educación del Virreinato a la época contemporánea". En: Manual de Historia de Colombia. Tomo III. Bogotá: Colcultura.

LOBATO, Mirta. (dir.). (2010). Nueva historia Argentina. El progreso, la modernización y sus límites (1880-1916). Buenos Aires: Editorial Sudamericana.

OSSENBACH, Gabriela. (2001). "Génesis histórica de los sistemas educativos". En: Organización de Estados Iberoamericanos. Cuadernos de Educación Comparada. Génesis, estructura y tendencias de los sistemas educativos iberoamericanos. Madrid: OEI.

PIÑERES, Dora. (2008). Modernización, Universidad y Región. El caso de la Universidad de Cartagena, 1920-1946. Cartagena: Universidad de Cartagena, Universidad Pedagógica y Tecnológica de Colombia (UPTC), RUDECOLOMBIA.

REVISTA HISTORIA DE LA EDUCACIÓN LATINOAMERICANA (RHELA). Vol. $20 . \quad N^{\circ} 30$. Enero - junio de 2018. Universidad Pedagógica y Tecnológica de Colombia: Tunja.

SERrANO, Sol., PONCE DE LEÓN, Macarena y RENGIFO, Francisca. (2013). Historia de la educación en Chile (1810-2010), Tomo I y II. Santiago de Chile: Taurus.

TANCK DE ESTRADA, Dorothy. (2010). Historia mínima. La educación en México. México: El Colegio de México.

URREGO, Miguel. (2005). La Revolución en Marcha de Colombia (1934-1938). Una lectura en perspectiva latinoamericana, Morelia: Universidad de Michoacán de Nicolás de Hidalgo-Revista Nueva Gaceta. 